\title{
DNA aptamer configuration affects the sensitivity and binding kinetics of thrombin
}

\author{
Alexandra Poturnayováa , Maja Šnejdárkováa, Tibor Hianik \\ ${ }^{a}$ Institute of Biochemistry and Animal Genetics, Slovak Academy of Sciences, Ivanka pri Dunaji, Slovakia \\ ${ }^{b}$ Faculty of Mathematics, Physics and Informatics, Comenius University, Bratislava, Slovakia \\ alexandrapoturnayova@gmail.com, alexandra.poturnayova@savba.sk
}

\begin{abstract}
Thrombin is serine protease involved in the coagulation cascade, which converts soluble fibrinogen into insoluble strands of fibrin - a matrix of the blood clot formation. Development of the sensitive method of the thrombin detection in nanomolar level is important for clinical practice. In this work we applied acoustic thickness shear mode method (TSM) for study the binding of human thrombin depending on DNA aptamer configuration. We compared sensitivity of detection and binding kinetics of the thrombin to the conventional DNA aptamers and aptamer dimers immobilized at the surface of quartz crystal transducer. We have shown that aptasensors based on aptamer dimers more sensitively detect thrombin. The aptamer-thrombin complexes were also more stable as revealed from equilibrium dissociation constant, $K_{D}$, that was 4 times lower for aptamer dimers in comparison with conventional aptamers. Determination of motional resistance, $R_{m}$, from acoustic impedance analysis allowed us to find important differences in physico-chemical properties of layers formed by conventional aptamers and aptamer dimers.
\end{abstract}

Keywords: DNA aptamers, Aptamer dimers, Human thrombin, Thickness shear mode, Biosensor

\section{Introduction}

The monitoring of the biorecognition processes and detection of molecules play an essential role in the basic research as well as in clinical practice. The DNA aptamers are single stranded oligonucleotides containing 25-80 bases that can be selected in vitro by SELEX (Systematic Evolution of Ligands by EXponential enrichment) method (Ellington and Szotak 1990, Tuerk and Gold 1990). Due to the specific structure aptamers can recognize their target with high specificity similar to that of antibodies (Jayasena 1999, Ellington and Szotak 1990, Tuerk and Gold 1990). Efficiency of DNA aptamers for detection proteins has been demonstrated in several works focused on development label-free biosensors for detection: of immunoglobulin $\mathrm{E}$ (Liss et al. 2002, Proske et al. 2002, Maehashi et al. 2007, Šnejdárková et al. 2008), cellular prions (Hianik et al. 2009a), human thrombin (Hianik et al. 2005, Cai et al. 2006) or other molecules (see Cheng et al. (2009) for review). The binding of ligands to aptamers depends on their 3D structure (Wang et al. 1993, Macaya et al. 1993, Chou et al. 2005). The proper folding of aptamers at the solid support may depend on the method of their immobilization. The method based on attachment of biotinylated aptamers to a gold support with chemisorbed neutravidin is rather convenient and well approved (Hianik 2009).

The DNA aptamers sensitive to thrombin are among most detailed studied. Thrombin is a serine protease that has many effects on the coagulation mechanism (Fenton and Ann 1981, Mann et al. 1990). The activation of thrombin is a crucial process in physiological and pathological coagulation and various rare diseases. For example, blood from a ruptured cerebral aneurysm clots around a cerebral artery and releases thrombin, which can induce an acute and prolonged narrowing of the blood vessel, potentially resulting in cerebral ischemia and infarction (Diculescu et al. 2011). Thrombin converts soluble fibrinogen to insoluble fibrin that forms the fibrin gel, which is responsible either for a physiological plug or for pathological thrombus (Holland et al. 2000). The positively charged fibrinogen-binding site at thrombin is responsible for this process, while heparin-binding site controls the anticoagulant function. These binding sites are spatially separated and localized at opposite poles of the thrombin (Hasegawa et al. 2008).

The first aptamer sensitive to fibrinogen binding site at thrombin (TBA) was developed by Bock et al. (1992). TBA is composed of 15 nucleotides 5'-(GGT TGG TGT GGT TGG)-3' and at certain ionic composition or in the presence of thrombin forms guanine quadruplex containing specific binding site. This aptamer has been developed with a purpose of thrombin inhibition for prevention of thrombosis. Now, it is also widely used as a receptor for thrombin detection in biosensors. Recently, we showed that aptamer dimer formed by two identical aptamers sensitive to fibrinogen binding sites are about twice more effective in thrombin inhibition in comparison with conventional aptamers (Poniková et al. 2011). In contrast, the 
sensitivity of aptamer homodimers immobilized at surfaces to the thrombin revealed higher (Hianik et al. 2008) or comparable (Hianik et al. 2009b) binding properties to thrombin depending on the method of aptamer immobilization. Our recent AFM (Neundlinger et al. 2011) and electrophoresis (Poniková et al. 2011) studies showed that aptamer homodimer maintains two binding sites, but due to close distance between guanine quadruplexes, the binding sites to thrombin could be disturbed. In this work we, therefore, further investigated the binding of thrombin to conventional aptamers and to the aptamer homodimers and used for this purpose thickness shear mode acoustic method.

\section{Materials and methods}

\section{Reagents}

All chemicals were of the highest purity commercially available: thrombin from human plasma was supplied by Sigma - Aldrich (Germany), neutravidin was purchased from Pierce (USA). Inorganic salts (p.a.), chloroform, acetone, ethanol, methanol were purchased from Slavus (Slovakia). The highly purified deionized water (ELIX 5, Millipore, USA) was used in all experiments.

We used 30-mer DNA aptamer 5'- GGT TGG TGT GGT TGG TTT TTT TTT TTT TTT - 3' (BFTT) contained 15-mer dT spacer modified at $5^{\prime}$ end by biotin and 35-mer DNA aptamer 5'- AAA AAA AAA AAA AAA TTT TTG GTT GGT GTG GTT GG - 3' (FAT2), contained 15-mer dA spacer complementary to the spacer at BFTT. Both aptamers have typical motif of oligonucleotides GGT TGG TGT GGT TGG with affinity to the fibrinogen binding sites of the thrombin. The aptamers were purchased from Thermo Fischer Scientific GmbH (Germany). The mixture of aptamers BFTT+FAT2 were prepared by dimerization of their complementary supporting part. For this purpose the 1:1 mixture of both aptamers of the same concentration $\left(1 \mu\right.$ mol.L ${ }^{-1}$ BFTT: $1 \mu$ mol.L ${ }^{-1}$ FAT2) was placed in Eppendorf tube. The mixture was heated up to $95^{\circ} \mathrm{C}$ and then allowed to cool at ambient temperature $\left(25^{\circ} \mathrm{C}\right)$. This process ensures formation of proper DNA duplex of aptamers supporting part as well as formation of guanine quadruplexes.

\section{The acoustic thickness shear mode method}

The acoustic properties of quartz transducer have been studied by the thickness shear mode method (TSM). This is a well-established tool for mass and surface viscosity-sensitive detection of affinity interaction at a quartz crystal transducer surface. This method allowed us to analyze the changes in series resonant frequency $f_{s}$ and motional resistance $R_{m}$ of a quartz crystal. The shift in resonant frequency of the crystal indicates the changes of its mass. The motional resistance is sensitive to the viscoelastic properties of the biolayer and to the interaction with surrounding solvent (Holloway et al. 2003, Ellis and Thompson 2004, Ellis and Thompson 2010). A 8712AS network analyzer (Agilent Technologies, USA) was used to measure the impedance spectra of the sensor, from which the values $f_{s}$ and $R_{m}$ were determined. The changes of frequency and motional resistance were studied in flow-through cell (Cavic et al. 1999). The apparatus is described in detail elsewhere (Šnejdárková et al. 2008).

\section{Preparation of aptasensors}

For the preparation of the layer we used polished AT-cut quartz with a fundamental frequency of $8 \mathrm{MHz}$ (CH Instruments, USA), covered on both side by gold electrodes (working area $0.2 \mathrm{~cm}^{2}$ ). The crystal was cleaned in ultrasound bath type sonicator DT-31 (Bandelin Electronic, Germany) during $30 \mathrm{~min}$ in $0.1 \%$ sodium dodecyl sulphate, then $15 \mathrm{~min}$ in Millipore water, $5 \mathrm{~min}$ in: acetone, ethanol and methanol. Then the crystal was washed with ethanol and dried under nitrogen stream. After this step the crystal was mounted between two silicon o-rings in the flow-through cell (cell was a gift of prof. M. Thompson, University of Toronto, Canada) in such a way that the analyte solution was added only at one side of the crystal. The effective volume of the cell was $100 \mu \mathrm{l}$. The buffer and analyte were introduced into the cell by means of a Genie Plus syringe pump (Kent Scientific, USA).

Neutravidin (NA) was dissolved in Millipore water in a concentration $250 \mu \mathrm{g} \cdot \mathrm{mL}^{-1}$. The solution of NA was allowed to flow in the cell with a rate $50 \mu \mathrm{l} . \mathrm{min}^{-1}$ for $20 \mathrm{~min}$. Thistime was sufficientfor reaching saturation in coverage of the crystal surface by chemisorption as observed by measurement of resonant frequency and motional resistance. Then, the Millipore water was added to flow in order to remove physically adsorbed NA. Finally, $2 \mu \mathrm{mol} . \mathrm{L}^{-1}$ aptamer BFTT or homodimer $1 \mu \mathrm{mol.L} \mathrm{L}^{-1}$ BFTT-FAT2 in thrombin/binding buffer (TRB) (140 mmol.L $\mathrm{L}^{-1} \mathrm{NaCl}, 5 \mathrm{mmol} . \mathrm{L}^{-1} \mathrm{KCl}$, $1 \mathrm{mmol} . \mathrm{L}^{-1} \mathrm{CaCl}_{2} \cdot 2 \mathrm{H}_{2} \mathrm{O}, 1 \mathrm{mmol} . \mathrm{L}^{-1} \mathrm{MgCl}_{2} \cdot 6 \mathrm{H}_{2} \mathrm{O}$, 20 mmol.L - $^{-1}$ TRIS, pH 7.4) were allowed to flow in the cell for $20 \mathrm{~min}$. After establishment the steadystate conditions the thrombin dissolved in TRB (concentrations 30, 100, 200 and 300 nmol.L ${ }^{-1}$ ) was allowed to flow in the cell and the $f_{s}$ and $R_{m}$ values were measured continuously. After each addition of thrombin and establishment of steady-state conditions the sensor surface was washed by flow of the TRB in order to remove not specifically bound thrombin. 


\section{Results and Discussion}

In this work we studied the interaction of human thrombin with DNA aptamers immobilised on a quartz crystal transducer covered by neutravidin (NA). We compared the interactions of thrombin with DNA aptamers of two configurations - conventional single stranded aptamers (BFTT) and aptamer dimers containing two identical binding sites (BFTT-FAT2), respectively. The results of binding events were expressed as the changes of series resonant frequency $f_{s}$ and motional resistance $R_{m}$. We should note that similar aptamers with slightly different spacers were studied earlier by us (Hianik et al. 2009b). However, in the present work we focused in more detail on the formation of the sensing surface, role of the aptamer density in sensitivity of thrombin detection and on the effect of aptamer configuration on the changes in motional resistance.

The changes of $f_{s}$ and $R_{m}$ values during the formation of the NA layer and adsorbtion of BFTT or BFTT-FAT2 aptamers as a function of time are presented in Fig. 1A and B, respectively. Addition of NA dissolved in the water resulted in a sharp decrease of resonant frequency in average by $180 \mathrm{~Hz}$, but $R_{m}$ values practically did not change. This is in agreement with previously reported data (Tassew and Thompson 2003). The decrease of resonant frequency is evidence of a strong adsorption of NA to a gold surface, but negligible changes in $R_{m}$ suggests formation of a rigid protein layer. Washing of the surface by deionised water caused certain increase of resonant frequency, which is due to removal of physically adsorbed NA molecules. Because aptamers were dissolved in TRB buffer, the sensor was washed with this buffer prior addition of aptamers in order to obtain proper base line. This washing resulted in sharp decrease of both resonant frequency and motional resistance, which is due to changes in ionic strength that affect the molecular slip between biolayer and the buffer (Sakti et al. 2001, Ellis and Thompson 2004). The new baseline was achieved after 15 min following starting the TRB flow. Addition of $2 \mu \mathrm{mol} . \mathrm{L}^{-1}$ BFTT resulted in decrease of resonant frequency and in increase of motional resistance (Fig. 1A). The steady-state values after washing the surface with TRB buffer were $49 \mathrm{~Hz}$ and $1.3 \Omega$, respectively. The decrease of resonant frequency is due to binding of biotinylated DNA aptamers to NA layer, while increase of $R_{m}$ value suggests increased contribution of viscosity, probably due to stronger interaction of flexible oligonucleotides with the buffer. This agrees well with previously published data (Hianik et al. 2009b). Addition of aptamer homodimers BFTT-FAT2 also resulted in decrease of resonant frequency and in increase of motional resistance (Fig. 1B). However, decrease of resonant frequency $\left(\Delta f_{s}=111 \mathrm{~Hz}\right)$ was approx. two fold higher in comparison with that of conventional BFTT aptamers, which reflect two fold larger molecular weight of aptamer dimers. Surprisingly, also the increase of motional resistance $\left(\Delta R_{m}=6.2 \Omega\right)$ was substantially (4.8 times) larger for aptamer dimers in comparison with conventional aptamers. It is likely, that due to rigid supporting part and improved orientation of aptamer dimers at the surface the water molecules more easily entrapping into the DNA layer. This makes the interaction between water and oligonucleotides more extensive and hence caused larger contribution to the surface viscosity.
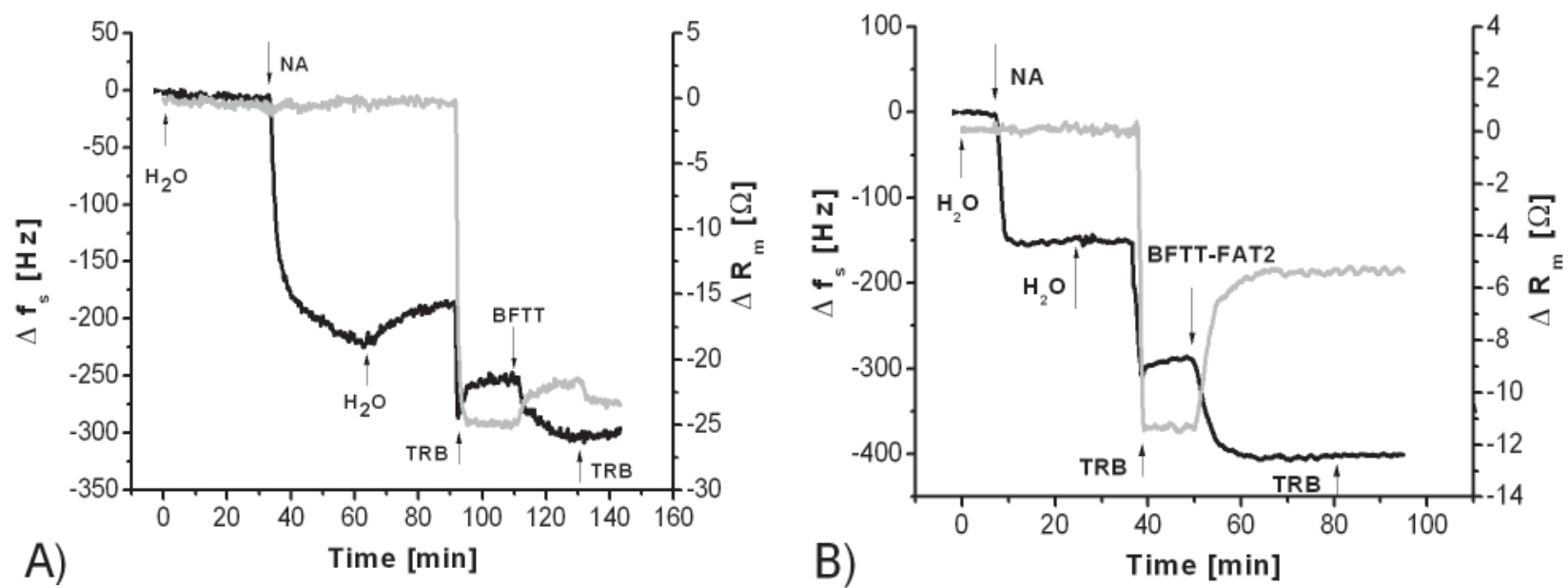

Fig. 1. Representative plot of the kinetics changes of resonant frequency, $\Delta f_{s}$ (black), and motional resistance, $\Delta R_{m}$ (gray), following various modifications of quartz crystal surface: A: NA and aptamer BFTT; B: NA and homodimer BFTT-FAT2. Addition of the NA, aptamers and washing the surface by water or TRB buffer is marked by arrows. 
The response of the biosensors based on conventional, BFTT aptamers and aptamer dimers (BFTTFAT2) following addition of various thrombin concentrations is compared on Fig. 2 where the plot of resonant frequency $(\mathrm{A})$ and motional resistance (B) as a function of thrombin concentration is presented. It can be seen that already at relatively low concentration of thrombin $\left(30 \mathrm{nmol} . \mathrm{L}^{-1}\right)$ rather large decrease of resonant frequency takes place. These changes are substantially larger for aptasensor based on homodimers in comparison with that for conventional aptamers. This is probably due to larger number of binding sites for aptasensor based on dimers. However, when twice-larger surface density of aptamer dimers was used $\left(2 \mu\right.$ mol.L ${ }^{-1}$ BFTTFAT2) we did not observe changes in frequency and in motional resistance. Most probably, higher aptamer density caused sterical hindrance that does not allowing proper formation of guanine quadruplexes and hence formation of thrombin-binding sites. With increasing concentration of thrombin the saturation in frequency changes took place. We observed also substantial differences in changes of motional resistance as a function of thrombin concentration for aptasensors based on conventional aptamers and aptamer dimers (Fig. 2B).

As it can be seen from Fig. 2B for conventional aptamers the motional resistance sharply decreases already at relatively low thrombin concentration (30 nmol.L ${ }^{-1}$ ) and reaches saturation at the concentrations above $100 \mathrm{nmol} . \mathrm{L}^{-1}$ of thrombin. In contrast, for aptasensors based on aptamer dimers the changes in motional resistance were much less expressed. After initial small decrease the $R_{m}$ value slightly increased and reached saturation at
300 nmol. $\mathrm{L}^{-1}$ of thrombin. These differences are most likely due to different structural and physicochemical properties of the aptamer layers. As we mentioned above, the layer composed of aptamer dimers allowing entrapment of additional water molecules into the biolayer, which substantially affect the surface viscosity. Adsorption of rigid thrombin molecules to such a surface probably does not affect the layer rigidity and hence also the surface viscosity. In contrast, the layers composed of conventional DNA aptamers can be more randomly ordered due to flexible oligonucleotide chains. Addition of thrombin may induce formation of more rigid oligonucleotide layer, which can explain the decrease of motional resistance. However, further analysis is required for understanding these structural and physical changes, which is out of the scope of this work. We should also mention that in control experiments we studied the interaction of thrombin with only NA layer, without aptamers, and no detectable changes both in resonant frequency and motional resistance were observed (data are not shown).

In order to analyze quantitatively the kinetics of the binding of the thrombin to the conventional aptamers and aptamer dimers we determined the kinetics constants of the binding reaction. The analysis was based on known peculiarities of binding of the ligands to the receptors (Skládal and Horáček 1999, Skládal 2003). The scheme of the binding of the ligand (thrombin) to the receptor (aptamer) immobilized at the surface is shown on Fig. 3. The binding of the ligand to the receptor is characterized by association, $k_{a}$, and dissociation, $k_{d}$, kinetic constants. Having these constants, it is pos-

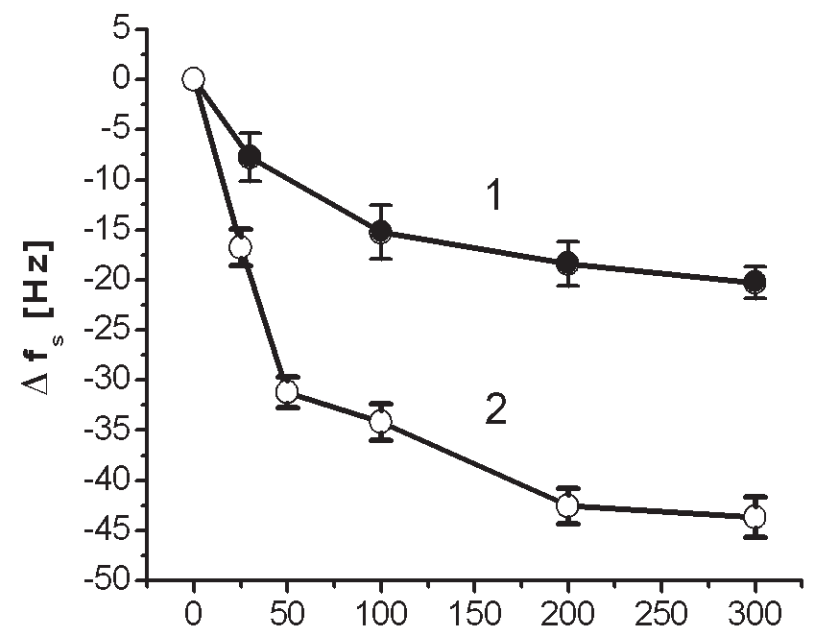

A) Thrombin [nmol. $\left.\mathrm{L}^{-1}\right]$

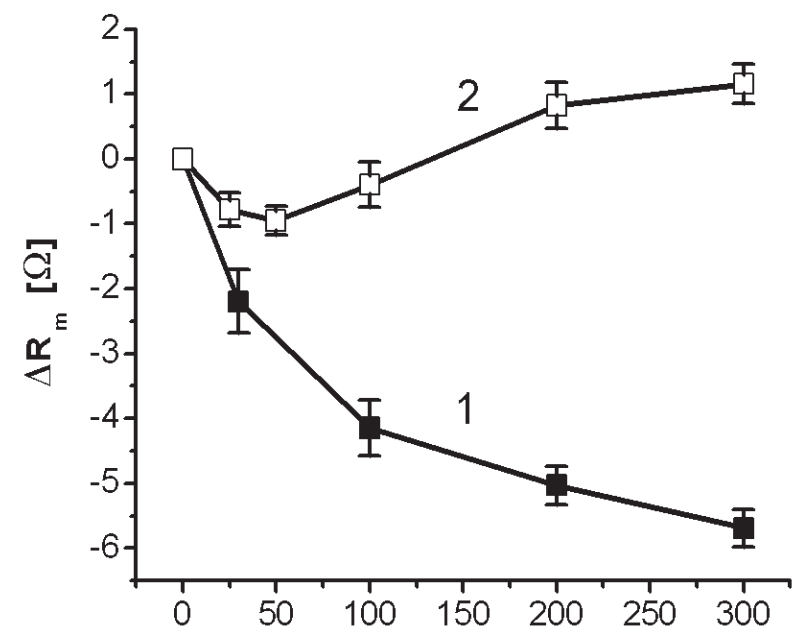

Thrombin [nmol.L $\left.{ }^{-1}\right]$

Fig. 2. The plot of the changes of resonant frequency (A) and motional resistance (B) as a function of thrombin concentration for aptasensor based on: BFTT (1) and BFTT-FAT2 (2) aptamers.

The results represent mean \pm S.D obtained in three independent experiments. 
sible to determine also the equilibrium constants of association and dissociation, respectively: $K_{A}=k_{a} / k_{d}$, $K_{D}=1 / K_{A}$ (Skládal and Horáček 1999).

$$
-A+B \rightleftharpoons k_{a} \rightleftharpoons-A B
$$

Fig. 3. The scheme of the binding of ligand B (thrombin) to its receptor A (aptamer), that is immobilized at the solid support, $k_{a}$ and $k_{d}$ are association and dissociation kinetic constants

(Skládal and Horáček 1999, Skládal 2003).

The kinetics and equilibrium constants were determined according to the procedure described in reference Skládal and Horáček (1999) or Skládal (2003). These constants that characterize binding of thrombin to the conventional aptamers (BFTT) and aptamer dimers (BFTT-FAT2) are summarized in Table 1 . The rate constants $k_{a}$ and $k_{d}$ and equilibrium constants $K_{A}, K_{D}$ for conventional aptamers substantially differ from that of aptamer dimers. For example the $K_{D}$ value for conventional aptamers was $(36.6 \pm 3.4)$ nmol. $\mathrm{L}^{-1}$, which is higher in comparison with that for aptamer dimers: $(8.7 \pm$ 2.7) nmol. $\mathrm{L}^{-1}$ and is in good agreement with previously reported data (Hianik et al. 2009b). Thus means that complex BFTT-thrombin is less stable in comparison with that based on aptamer dimers BFTT-FAT2. (Hianik et al. 2009a, Hasegawa et al. 2008).

Thus, the TSM method allowed to obtain important information on the physical and binding properties of aptasensors composed of aptamers of different configurations. Important conclusion from above study consists in necessity to select optimal density of aptamers at the surface.

\section{Conclusion}

In this work we used thickness shear mode acoustic method to study the interaction between thrombin and DNA aptamers immobilized on the surface of quartz crystal transducer. The immobilization of the aptamers to the surface and their configuration is crucial for sensor sensitivity. Therefore we com- pared sensitivity of thrombin detection by two types of aptasensors composed of conventional single stranded aptamer (BFTT) and by aptamer dimers (BFTT-FAT2) and showed that the sensor based on aptamer dimers is substantially more sensitive in thrombin detection. In order to analyze quantitatively the kinetics of the binding of thrombin to these aptasensors we determined also kinetics and equilibrium binding constants. The equilibrium constant of dissociation, $K_{D}$, for BFTT and BFTTFAT2 were $(36.6 \pm 3.4)$ nmol.L $\mathrm{L}^{-1}$ and $(8.7 \pm 2.7)$ nmol.L $L^{-1}$, respectively. This means that complex BFTT-thrombin is less stable in comparison with that based on aptamer dimers. Comparison of motional resistance, $R_{m}$, during binding of aptamers and thrombin suggests different physico-chemical properties of the layers formed by conventional aptamers and aptamer dimers.

\section{Acknowledgements}

This work was supported by Slovak Academy of Sciences MNT-era.NET II ID431, grant agreement No 234989 (M. Š. ), Agency for Promotion Research and Development under the project number APVV 0410-10 and Grant agency VEGA 1/0785/12. This publication is also the result of the project implementation: BIOMAKRO2, ITMS: 26240120027, supported by the Research E Development Operational Programme funded by the ERDF (T. H.).

\section{References}

Bock LC, Griffin LC, Latham JA, Vermaas EH, Toole JJ (1992) Nature 355: 564-566.

Cai H, Ming-Hung T, Hsing IM (2006) Sensors and Actuators B 114: 433-437.

Cavic BA, Hayward GL, Thompson M (1999) Analyst 124: 1405-1420.

Cheng AKH, Sen D, Yu H-Z (2009) Bioelectrochemistry 77: $1-12$.

Chou SH, Chin KH, Wang AHJ (2005) Trends in Biochemical Sciences 30 (5): 231-234.

Diculescu VC, Chiorcea-Paquim AM, Eritja R, OliveiraBrett AM (2011) Journal of Electroanalytical Chemistry 656: 159-166.

Ellington AD, Szostak J (1990) Nature 346: 818-822.

Ellis JS, Thompson M (2004) Chem. Commun.: 1310-1311.

Tab. 1. The kinetics constants of association, $k_{a}$, and dissociation, $k_{d}$, and equilibrium constants of association, $K_{A}$, and dissociation, $K_{D}$, for the interaction of the thrombin with conventional aptamers BFFT and aptamer dimers BFFT-FAT2 immobilized at the surface of neutravidin layers.

\begin{tabular}{|c|c|c|c|c|}
\hline Aptamer & $\begin{array}{c}\mathrm{k}_{\mathrm{a}} \\
10^{5} \mathrm{~mol}^{-1} \cdot \mathrm{L} \mathrm{s}^{-1}\end{array}$ & $\begin{array}{c}\mathrm{k}_{\mathrm{d}} \\
10^{-3} \mathrm{~s}^{-1}\end{array}$ & $\begin{array}{c}\mathrm{K}_{\mathrm{A}} \\
10^{7} \mathrm{~mol}^{-1} \cdot \mathrm{L}\end{array}$ & $\begin{array}{c}\mathrm{K}_{\mathrm{D}} \\
10^{-9} \text { mol. } \mathrm{L}^{-1}\end{array}$ \\
\hline BFTT & $6.78 \pm 1.79$ & $24.8 \pm 2.3$ & $2.73 \pm 0.25$ & $36.6 \pm 3.4$ \\
\hline BFTT-FAT2 & $0.30 \pm 0.08$ & $0.26 \pm 0.04$ & $11.54 \pm 3.6$ & $8.7 \pm 2.7$ \\
\hline
\end{tabular}


Ellis JS, Thompson M (2010) Langmuir 26 (13): $11558-11567$.

Fenton JW, Ann NY (1981) Acad. Sci 370: 468-495.

Hasegawa H, Taira K, Sode K, Ikebukuro K (2008) Sensors 8: 1090-1098.

Hianik T, Ostatná V, Zajacová Z, Stoiková E, Evtugyn, G (2005) Bioorganic \& Medicinal Chemistry Letters 15: 291-295.

Hianik T (2009). In: Mascini M (Ed) Aptamer in bioanalysis 5 (101-128). John Wiley \& Sons Inc., Hoboken, New Jersey.

Hianik T, Porfirieva A, Grman I, Evtugyn G (2008) Protein and Peptide Letters 15: 799-805.

Hianik T, Porfirieva A, Grman I, Evtugyn G (2009a) Protein and Peptide Letters 16: 363-367.

Hianik T, Grman I, Karpišová I (2009b) Chem. Commun. 41: 6303-6305.

Holland CA, Henry AT, Whinna HC, Church FC (2000) FEBS Letters 484: 87-91.

Holloway AF, Nabok A, Thompson M, Ray AK, Crowther D, Siddiqi J (2003) Sensors 3: 187-191.

Jayasena SD (1999) Clinical Chemistry 45: 1628-1650.

Liss M, Peterson B, Wolf H, Prohaska E (2002) Anal. Chem. 74: 4488-4495.

Macaya RF, Schultze P, Smith FW, Roe JA, Feigon J (1993) Proc. Sci. U.S.A. 90: 3745-3749.
Maehashi K, Katsura T, Kerman K, Takamura Y, Matsumoto K, Tamiya E (2007) Anal. Chem. 79: $782-787$.

Mann KG, Nesheim ME, Church WR, Haley WR, Krishnaswamy S (1990) Blood 76: 1-16.

Neundlinger I, Poturnayová A, Karpišová I, Rankl C, Hinterdorfer P, Šnejdárková M, Hianik T, Ebner A (2011) Biophys. J. 101: 1781-1787.

Poniková S, Tlučková K, Antalík M, Víglaský V, Hianik T (2011) Biophys. Chem. 155, 29-35.

Proske D, Gilch S, Wopfner F, Schatzl HM, Winnacker EL, Famulok M (2002) Chem. Bio. Chem. 3: 717-725.

Sakti SP, Lucklum R, Hauptmann P, Buhling F, Ansorge S (2001) Biosensors and Bioelectronics 16: 1101-1108.

Skládal P, Horáček J (1999) Anal. Lett. 32: 1519-1529.

Skládal P (2003) J. Braz. Chem. Soc. 14: 491-502.

Šnejdárková M, Svobodová L, Polohová V, Hianik T (2008): Anal. Bioanal. Chem. 390: 1087-1091.

Tassew N, Thompson M (2003) Biophysical Chemistry 106: 241-252.

Tuerk C, Gold L (1990) Science 249: 505-510.

Wang KY, McCurdy S, Shea RG, Swaminathan S, Bolton PH (1993) Biochemistry 32: 1899-1904. 